\title{
A report on strategies for motivating and developing the computational thinking for and by women
}

Original Title: Um relato sobre estratégias de motivação e desenvolvimento do pensamento computacional para e por mulheres ${ }^{1}$

\section{Camila Sass ${ }^{\mathrm{a}}$, Julia Baldia, Lara Tenore ${ }^{a}$, Carla Rodriguez ${ }^{\mathrm{b}}$, Denise Goya ${ }^{\mathrm{b}}$, Mirtha Venero $^{\text {b }}$, Rafaela V. Rocha ${ }^{b}$}

${ }^{a}$ Bacharelado em Ciência e Tecnologia, Universidade Federal do ABC (UFABC) - Santo André, SP - Brasil

${ }^{\text {b }}$ Centro de Matemática, Computação e Cognição (CMCC), UFABC - Santo André, SP - Brasil

\section{ARTICLE INFO}

Article history:

Received 25 February 2019

Accepted 07 April 2020

Available online 05 May 2020

Keywords:

Gender

Computational thinking

Programming Teaching and

Learning

Higher Education

Palavras Chave:

Gênero

Pensamento Computacional

Ensino e Aprendizagem de

Programação

Educação Superior

ISSN: 2595-9077

DOI: JCThink.v3.n1.p34

\section{ABSTRACT}

INTRODUCTION: Initiatives to engage girls and women in STEM-related areas have been successfully reported in the literature. However, such initiatives should involve the participation of the local community and address their needs and interests. OBJECTIVE: This article reports a project that aims to apply different strategies to motivate and teach computational thinking for women, led by students of Science and Technology at UFABC. METHOD: The methodology proposes three working branches: research (understanding the factors that discourage girls from the Computer Science course), outreach actions and teaching programming logic for women. RESULTS: Preliminary research revealed a female participation of less than $18 \%$ in all subjects of the course and a percentage lower than $15 \%$ of female graduates. The teaching actions had a total of 61 graduates. The events held (workshop and seminar) had about 80 participants. CONCLUSION: The actions of research, teaching, extension and dissemination of this project managed to engage more than 200 women and contemplate the interests of the internal and external community to UFABC. As future work, the initiatives will be extended to high schools in the region. The methodology should be consolidated and applied at the institutional level to include other STEM courses at UFABC.

\section{RESUMO}

INTRODUÇÃO: Iniciativas para engajar meninas e mulheres nas áreas relacionadas a STEM têm sido reportadas com sucesso na literatura. Entretanto, essas iniciativas devem contemplar a participação da comunidade local e abordar suas necessidades e interesses. OBJETIVO: Este artigo relata um projeto que visa aplicar diferentes estratégias para motivar e ensinar o pensamento computacional para mulheres, liderado por alunas de Ciência e Tecnologia da UFABC. MÉTODO: A metodologia propõe três frentes de trabalho: pesquisa (compreensão dos fatores que afastam meninas do curso de Ciência da Computação), ações de divulgação e ensino de lógica de programação para mulheres. RESULTADOS: As pesquisas preliminares revelaram uma participação feminina menor de $18 \%$ em todas as disciplinas do curso e uma porcentagem inferior a $15 \%$ de alunas formadas. As ações de ensino tiveram o total de 61 concluintes. Os eventos realizados (workshop e seminário) tiveram cerca de 80 participantes. CONCLUSÃO: As ações de pesquisa, ensino, extensão e divulgação deste projeto conseguiram engajar mais de 200 mulheres e contemplar os interesses da comunidade interna e externa a UFABC. Como trabalho futuro, as iniciativas serão estendidas a escolas de ensino médio da região. A metodologia deve ser consolidada e aplicada no nível institucional para incluir outros cursos de STEM da UFABC.

\section{Introdução}

Segundo o relatório da UNESCO (2018) sobre educação de meninas e mulheres em STEM (Science, Technology, Engineering and Mathematics), as mulheres representam apenas $35 \%$ de todas as pessoas matriculadas na educação superior nos campos de estudo relacionados. A diferença quantitativa entre mulheres e homens em cursos de STEM é tão relevante mundialmente que é contemplada pela Agenda 2030 para o Desenvolvimento Sustentável da ONU; em particular, nos objetivos sobre educação

\footnotetext{
1 Versão estendida do artigo "Um Relato sobre Estratégias de Motivação e Ensino de Lógica de Programação para e por Mulheres" apresentado no WAlgProg 2018.
} 
inclusiva e equitativa de qualidade e aprendizagem ao longo da vida (ODS 4), e sobre a igualdade de gênero e empoderamento das meninas e mulheres (ODS 5). Como citado no relatório da UNESCO, os sistemas educacionais e as escolas exercem um papel importante em estimular o interesse das meninas em disciplinas de STEM, bem como em oferecer oportunidades para que elas acessem e se beneficiem de uma educação de qualidade na área.

Por isso, as iniciativas para engajar meninas e mulheres a começarem e permanecerem nessas carreiras devem ser promovidas nas escolas desde o ensino básico até o nível superior e na sociedade em geral. Sem ações que estimulem o aumento do número de mulheres nas carreiras relacionadas a STEM, as inovações na ciência e na tecnologia continuarão sendo guiadas pela visão e prioridades dos homens, os quais continuarão obtendo a maior parte das recompensas intelectuais e financeiras. Por exemplo, o relatório "Gênero no Panorama de Pesquisa Global da Editora Elsevier" (Elsevier, 2017), estudou a presença de homens e mulheres na pesquisa científica em 27 áreas e diversos países. Os resultados do relatório mostraram que as áreas como Engenharia, Ciência da Computação, Matemática e as Ciências Físicas têm menos de $30 \%$ de representação feminina. No Brasil, segundo a pesquisa realizada pelo Instituto Brasileiro de Geografia e Estatística (IBGE, 2018), desde 2012 as mulheres ganham cerca de $75 \%$ do que os homens recebem.

A Computação é uma das áreas das STEM de maior crescimento no mercado de trabalho e com pouca representatividade feminina. Até nas grandes empresas, como Google (2018), com políticas voltadas para aumentar a representatividade de gênero, a porcentagem de mulheres na área tecnológica é menor do que $22 \%$. Apesar das políticas implementadas pela Google, o crescimento da representatividade feminina nos dois últimos anos variou menos de $2 \%$ e ainda é um crescimento simbólico. Essa situação ilustra que mesmo quando as empresas buscam implementar políticas para aumentar a representatividade feminina, a baixa participação das mulheres nos cursos de ciência e tecnologia cria um grande desafio. Por isso, as Universidades devem ter um papel ativo e transformador para contribuir para a equidade de gênero nos cursos de STEM, fazendo a ponte entre o ensino básico e o mercado de trabalho.

Este artigo apresenta um projeto voltado para engajar meninas no curso de Ciência da Computação na Universidade Federal do ABC alinhado com os objetivos ODS 4 e 5. O projeto, nomeado ++C\&TpM: Desmistificando a Ciência da Computação e as Tecnologias da Informação por e para Mulheres ${ }^{2}$, nasceu de um grupo de mulheres com vontade de contribuir para que a igualdade de gênero seja alcançada nessa área. A equipe está composta por oito alunas protagonistas de todas as atividades e cinco docentes que ajudam na orientação e coordenação.

O projeto iniciou seus trabalhos no contexto de um edital para a melhoria do ensino, tendo como alvo meninas, com pouco ou nenhum contato com programação, que já haviam ingressado no ensino superior. Contudo, desde o início o grupo identificou como uma necessidade levar o conhecimento sobre Ciência da Computação para alunas de escolas públicas da região, para que elas possam ter contato com uma área que muito possivelmente desconhecem ou nem ao menos cogitam como uma possibilidade. $\mathrm{O}$ artigo descreve os primeiros resultados das ações realizadas para desenvolver o pensamento computacional e seu impacto em meninas ingressantes que não cogitaram uma carreira em Computação, ou sobre aquelas que apresentavam interesse, mas não se sentiam seguras em segui-la.

\footnotetext{
${ }^{2}$ http://pesquisa.ufabc.edu.br/lirte/ctpm/
} 
O artigo está organizado como segue. A seção 2 apresenta os trabalhos relacionados. Na seção 3 é apresentado o projeto ++C\&TpM e sua metodologia, seguida pelo desenvolvimento e resultados, na seção 4. A seção 5 discute estratégias para o engajamento de meninas da comunidade externa a UFABC. As conclusões e trabalhos futuros são apresentadas na seção 6 .

\section{Trabalhos Relacionados}

O Censo da Educação Superior 2016 do Instituto Nacional de Estudos e Pesquisas Educacionais Anísio Teixeira mostrou que, entre as oito grandes áreas dos cursos, a menor porcentagem de mulheres está nos cursos de Ciências, Matemática e Computação. Em particular, o curso de Ciência da Computação é o segundo com menor porcentagem de mulheres, após Engenharia Mecânica com cerca de 20\%. Os dados do relatório de estatísticas da Sociedade Brasileira de Computação (SBC 2017) apontam que a porcentagem de mulheres entre ingressantes, matriculados(as) e concluintes nos cursos da área não é maior que $16 \%$. Segundo a pesquisa realizada por Maia (2016), o de mulheres concluintes nos cursos de Computação decresce com pequenas oscilações.

Conscientes dessa baixa representatividade feminina, várias instituições governamentais e organizações da sociedade civil estão apoiando projetos para reverter esse cenário. Por exemplo, desde 2015 a SBC institucionalizou o Programa Meninas Digitais, criado em 2011 a partir de discussões no WIT (Women in Information Technology), evento satélite do Congresso da SBC. O programa tem como objetivo motivar alunas do ensino médio/tecnológico, ou nos anos finais do ensino fundamental, a desenvolver conhecimento nas áreas da Computação e Tecnologias da Informação e Comunicação (Maciel \& Bim, 2017). Ele inclui diversas ações como palestras, minicursos, oficinas e mais de 50 projetos parceiros no país.

O Fundo ELAS, Instituto Unibanco, Fundação Carlos Chagas e ONU Mulheres têm promovido projetos para estimular meninas a se envolverem com as ciências exatas e tecnológicas. Recentemente o Conselho Nacional de Desenvolvimento Científico e Tecnológico e o Ministério da Ciência, Tecnologia, Inovações e Comunicações lançaram a Chamada "Meninas nas Ciências Exatas, Engenharias e Computação", para desenvolver projetos entre escolas públicas da Educação Básica e Instituições de Ensino Superior, com o objetivo de aumentar a participação de meninas e mulheres nas áreas de STEM. Além disso, existem vários sites de caráter pessoal, idealizados e desenvolvidos por e para mulheres, e.g. PrograMaria, Reprograma, MinasProgramam, MariaLab, Mulheres na Computação.

A desigualdade de gênero nos cursos de Computação do Brasil também tem sido objeto de pesquisa em diversos eventos da área. O WIT tem promovido trabalhos com análises sobre a presença de mulheres em cursos no ensino superior brasileiro (Holanda et al., 2017; Matos et al. 2017; Monteiro et al., 2017; Nakamura et al., 2017; Nunes et al., 2016; Schmidt, Britto \& Koehler, 2016). O tema também têm sido discutido em outros congressos apoiados pela SBC, como o LAWCC (Congresso da Mulher Latinoamericana em Computação) (Bertoni e dos Santos, 2018; Bim, Figueiredo \& Maciel, 2107; da Silva et al., 2018; Freitas et al. 2017; Kozievitch et al., 2018; Monard \& Fortes, 2013) e o CBIE (Congresso Brasileiro de Informática na Educação) (dos Santos, 2017; Moreira et al., 2016; Posser \& Teixeira, 2016). No entanto, o cenário de gênero dentro das instituições de educação superior deve continuar sendo analisado, tanto quantitativa como qualitativamente, para compreender as causas e problemas locais e desenvolver ações que permitam aumentar a participação das mulheres (Grossi et al., 2016).

Dentre as causas dessa desigualdade estão os estereótipos de gênero, que retratam cientistas e engenheiros da computação como sendo, em sua maioria, homens brancos, 
com brilhantes habilidades matemáticas, socialmente isolados, competitivos e intensamente focados na tecnologia (Cheryan, Master \& Meltzoff, 2015; Lewis, Anderson \& Yashuhara, 2016; Margolis \& Fisher, 2003). Esses e outros estereótipos impactam às meninas desde a tenra idade através da mídia, os pais e professores, às vezes inconscientemente (Master, Cheryan \& Meltzoff, 2016; Reinking \& Martin, 2018). Por isso, a exposição a modelos e ambientes femininos positivos contribui a aumentar o interesse, a autoconfiança e o sentido de pertencimento das meninas nas áreas de STEM (Hughes, Nzekwe \& Molyneaux, 2013; Rosenthal et al. 2011; Sax et al., 2018). Os professores, os cursos introdutórios e as experiências dentro e fora da sala de aula são também importantes para incentivar as meninas a persistirem em carreiras STEM (Banerjee et al., 2018; Mervis, 2011). Por exemplo, atividades extracurriculares e ambientes informais de aprendizagem para meninas permitem explorar e aprender os conteúdos de STEM como hobby, sem o rigor acadêmico (Blaney \& Stout, 2017; Dasgupta \& Stout, 2014; Reinking \& Martin, 2018).

\section{O Projeto $++C \& T p M$ da UFABC e sua Metodologia}

A UFABC foi a primeira universidade brasileira a adotar um modelo pedagógico de ensino interdisciplinar onde todos os discentes ingressam por meio de Bacharelados Interdisciplinares (BI) (UFABC, 2017): o Bacharelado em Ciência e Tecnologia (BC\&T) e o Bacharelado em Ciências e Humanidades (BC\&H).

Diferente do sistema educacional tradicional, a estrutura da grade curricular da UFABC é baseada no regime quadrimestral e em um sistema de créditos que permite diferentes organizações curriculares, conforme os interesses das(os) discentes. Ou seja, cada aluna(o) é responsável por se matricular nas disciplinas que têm interesse. No entanto, existem disciplinas obrigatórias em comum para todas(os). Este sistema preserva a ideia de liberdade para a exploração de novos caminhos em todas as atividades acadêmicas, e nele as alunas e os alunos podem optar por um ou vários cursos específicos após o BC\&T ou BC\&H. O projeto pedagógico da UFABC permite moldar carreiras compatíveis com os avanços científicos, tecnológicos e sociais.

Apesar do seu modelo com foco na interdisciplinaridade, no diálogo entre todas as áreas do conhecimento e na flexibilidade curricular, o problema da desigualdade de gênero também está presente nas carreiras de STEM da UFABC. Segundo dados da PróReitoria de Planejamento e Desenvolvimento Institucional da UFABC (PROPLADI, 2017), a porcentagem de mulheres discentes na universidade cresceu de $31.4 \%$ em 2012 a $34.2 \%$ em 2016. No entanto, no BC\&T a porcentagem de meninas no campus Santo André (onde se concentram a maioria dos cursos pós-BC\&T) diminuiu de $30.8 \%$, em 2012, para $27.8 \%$ em 2016, enquanto no campus São Bernardo passou de $37.2 \%$ para $31.7 \%$.

Para determinar os fatores que influenciam a baixa representatividade feminina nas áreas de STEM da UFABC, os dados acima e outros devem ser analisados nos cursos pós-BI, em particular aqueles em que a proporção de mulheres é significativamente menor. Dentre esses cursos, destacam-se o Bacharelado em Ciência da Computação (BCC) e Engenharia de Instrumentação, Automação e Robótica (IAR), que estão entre os cinco bacharelados específicos mais pretendidos após o BC\&T (PROPLADI, 2017), porém são os dois com menor proporção de meninas: o BCC com aproximadamente $14 \%$ e IAR com aproximadamente $10 \%$.

Uma pesquisa realizada no Centro de Matemática, Computação e Cognição (CMCC) revelou que o BCC é o curso com maior número de estudantes matriculados e ao mesmo tempo com a menor porcentagem de mulheres, $18.9 \%$. Diante desse cenário e do desejo de alunas e professoras de aumentar a quantidade de mulheres no curso, surgiu 
em 2018 o projeto $++C \&$ TpM. O projeto iniciou por meio de um edital de melhoria de ensino, com o objetivo de promover iniciativas que contribuam para um número maior de meninas no BCC, bem como egressas da UFABC com habilidades para a resolução computacional de problemas. A equipe de elaboração foi composta por cinco professoras do BCC e na seleção das monitoras foram escolhidas oito alunas do BC\&T (três bolsistas e cinco voluntárias); cinco delas com pretensão pós-BI em Ciência da Computação, duas Engenharia Biomédica e uma Engenharia de Energia.

A metodologia do projeto baseou-se nos cinco pilares que são considerados de maior impacto para o interesse de meninas segundo a Microsoft Corp (2017): (i) modelos femininos reais a quem possam se identificar, (ii) experiências práticas, (iii) encorajamento e mentoria, (iv) compreensão das aplicações práticas e relevância para o futuro e (v) confiança que serão tratadas com igualdade na carreira. Com base nesses pilares, a equipe do projeto identificou a necessidade de protagonismo das alunas no projeto, que permitiria fornecer modelos femininos de sucesso na UFABC (Herrmann et al., 2018), bem como a quebra de estereótipos e a sensação de não pertencimento (Master, Cheryan \& Meltzoff, 2016). Na proposta foram definidas três frentes principais de trabalho:

- pesquisa e coleta de dados: sobre o tema das mulheres na tecnologia, dentro e fora da Universidade, para tentar compreender melhor o problema, identificar as melhores iniciativas práticas a serem desenvolvidas e analisar dados ao longo dos anos para avaliar o impacto das ações do projeto;

- divulgação em redes sociais e site: conteúdos com exemplos de mulheres que atuam e são muito importantes na área de computação, dúvidas sobre a carreira, ações do projeto, entre outras informações. O objetivo dessa frente é proporcionar maior divulgação sobre o tema e a carreira para o público feminino, de um ponto de vista feminino;

- ensino e extensão: desenvolvimento de habilidades de lógica de programação em meninas ingressantes na universidade. Esta frente foi motivada pela experiência das próprias alunas, pois o início do BCC pode ser bastante difícil para quem não teve contato com programação. Além disso, o desenvolvimento do pensamento computacional e suas habilidades (abstração, decomposição, reconhecimento de padrões, algoritmos) são importantes não só para cursos afins à Computação, mas também constituem mais um pilar na solução de problemas das Ciências Exatas e das Engenharias (Wing, 2006).

\section{Desenvolvimento e Resultados de Ações na Graduação}

A seguir são reportadas as atividades desenvolvidas e os resultados alcançados nas três frentes do projeto: Pesquisa (Seção 4.1), Divulgação (Seção 4.2) e Ensino (Seção 4.3). As ações de extensão envolvendo participantes externos a UFABC são apresentadas na Seção 5.

\subsection{Pesquisa}

Na primeira frente de trabalho do projeto, buscou-se avaliar possíveis causas que influenciam a baixa adesão de mulheres no curso de BCC. Um dos parâmetros analisados foi o desempenho acadêmico das discentes do sexo feminino nas disciplinas básicas (obrigatórias do $\mathrm{BC} \& \mathrm{~T}$ ), que lidam com o pensamento computacional e lógica de programação: Bases Computacionais da Ciência e Processamento da Informação. A primeira hipótese levantada era de que o baixo desempenho das discentes nessas disciplinas poderia levar a um desestímulo, fazendo com que elas desistissem de cursar 
posteriormente o BCC. Durante o 2016 e 2017, um total de 8449 estudantes cursaram essas disciplinas, com uma porcentagem de alunos do sexo masculino de aproximadamente $70 \%$. A Figura 1 ilustra o desempenho em ambas as matérias nesses anos, nos quais os conceitos de "A" a "D" representam aprovação e os conceitos " $F$ " $\mathrm{e}$ "O" reprovação por desempenho e frequência, respectivamente.

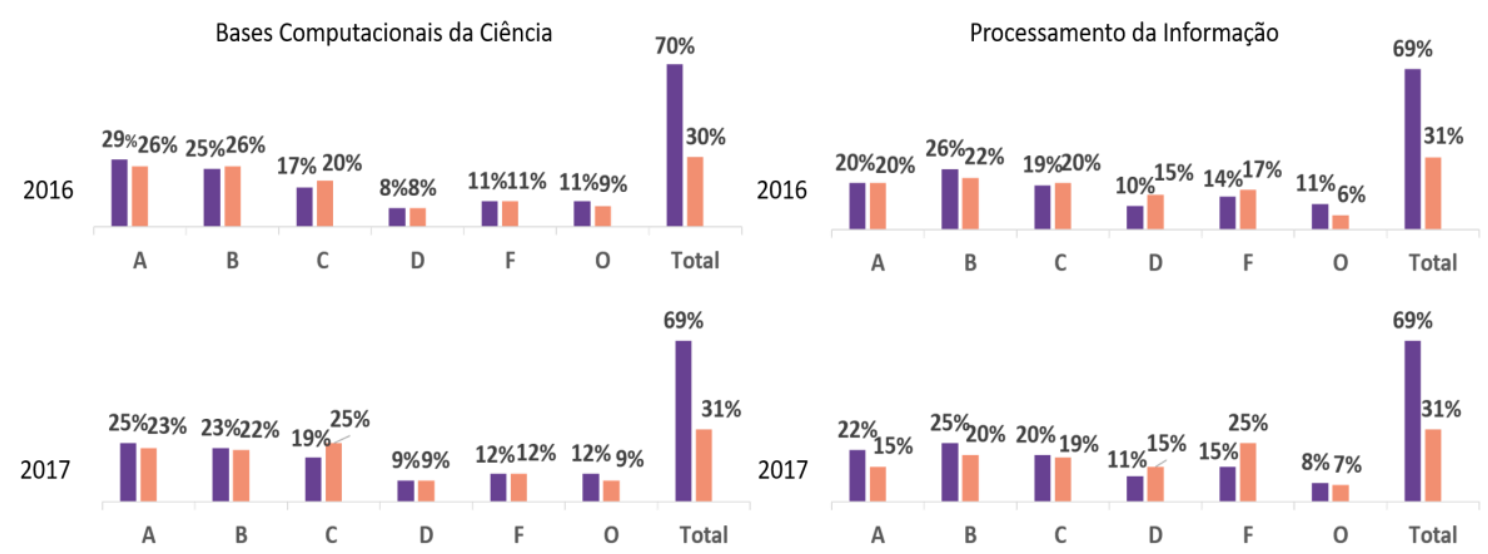

Figura 1. Desempenho de discentes nas disciplinas de Bases Computacionais da Ciência (esquerda) e Processamento da Informação (direita), por sexo (masculino em cor roxa), em 2016 e 2017. Fonte: Pró-Reitoria de Graduação UFABC.

Ao fazer a análise do desempenho por gênero nessas disciplinas usando o teste $\mathrm{t}$ de Student, não foi encontrada diferença estatística significativa nas turmas de Bases Computacionais da Ciência em 2016 e 2017. Já em Processamento da Informação, houve diferença significativa em 2016, em que as mulheres (M) obtiveram notas melhores (M $=2,233 \pm 0,061 ; \mathrm{H}=2,062 \pm 0,042 ; \mathrm{p}$-valor $=0.0264)$, e em 2017, ano em que os homens (H) tiveram melhor desempenho $(\mathrm{M}=1,710 \pm 0,061 ; \mathrm{H}=2,115 \pm 0,040$; p-valor < 0,0001). Com base nesses resultados, é possível afirmar que ambos os gêneros têm desempenhos semelhantes, o que descarta a hipótese anterior, visto que esse não seria um fator determinante para a baixa representatividade feminina no curso de BCC.

Apesar do desempenho similar ao dos homens, a maior parte das mulheres do BC\&T não faz o BCC por fatores como estereótipos de gênero e o próprio ambiente do curso ser predominantemente masculino. A análise dos dados de estudantes matriculadas(os) em disciplinas específicas da Computação, revelou que a presença de mulheres sempre foi inferior a $18 \%$. Também foi possível observar que, conforme a disciplina se torna mais avançada na grade do BCC, a porcentagem de mulheres na turma diminui e chega, por exemplo, a 9,5\% na disciplina obrigatória de Algoritmos e Estruturas de Dados II (média de 2015 a 2018), e a 0\% na disciplina optativa de Prática Avançada de Programação C, ofertada em 2016 com um total de 21 alunos.

A pesquisa também revelou uma baixa participação feminina nas colações de grau do BCC. Conforme é mostrado na Figura 2, a porcentagem total de graduadas (última coluna do gráfico) é de 14,6\% desde a primeira colação de grau em 2011. A situação torna-se ainda mais preocupante ao constatar a redução do número de formandas no curso desde 2016 e não ter nenhuma graduada em 2017. Além disso, observou-se que a baixa participação feminina também acontece no corpo docente, no qual somente $26,4 \%$ são mulheres. As porcentagens são similares na pós-graduação em Ciência da Computação com $15,7 \%$ de estudantes e $25 \%$ de docentes mulheres. 


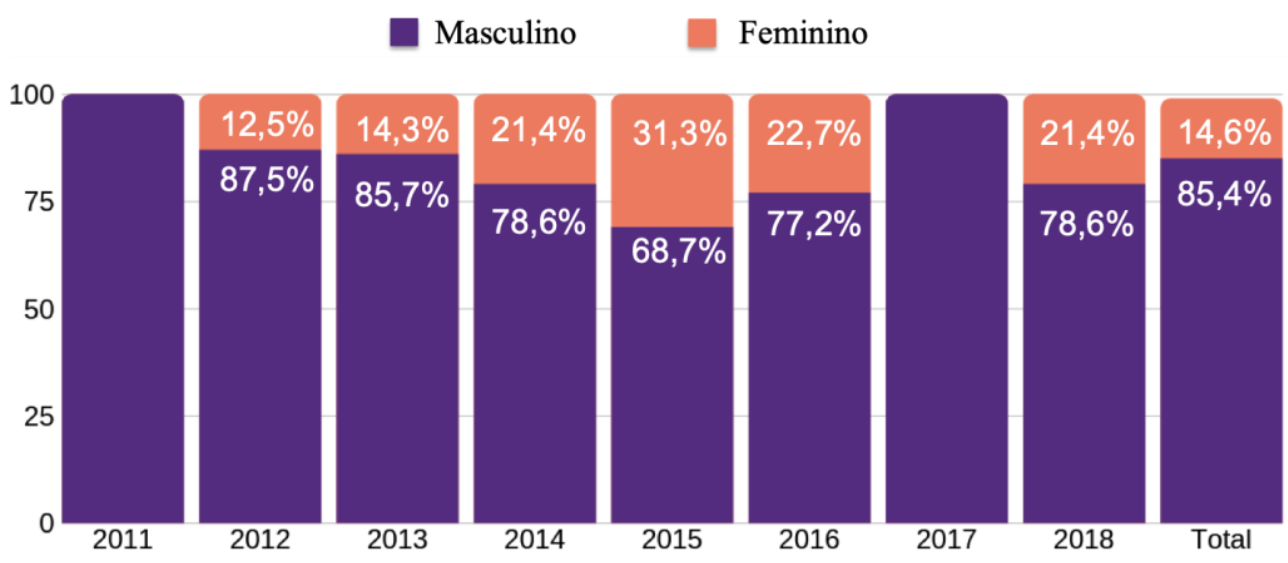

Figura 2. Participação por gênero nas colações de grau do BCC. Fonte: Secretaria acadêmica CMCC-UFABC.

$\mathrm{O}++\mathrm{C} \& \mathrm{TpM}$ tem como proposta investigar as possíveis causas que influenciam esse cenário, junto com profissionais da área de estudos de questões de gênero, formulando questionários e aplicando-os à comunidade acadêmica. Acredita-se que tendo conhecimento sobre os fatores que desestimulam as mulheres de cursar Ciência da Computação, é possível trabalhar melhor no desenvolvimento de iniciativas para atraí-las para a área e diminuir a evasão ao longo da graduação. Para isso, foi aplicado um questionário preliminar na comunidade acadêmica da UFABC em 2018. Foram obtidas 67 respostas, das quais 19,4\% são mulheres que pensam em cursar ou já estão cursando o BCC, contra 23,9\% de mulheres que não pretendem fazer o BCC (entre os homens, a proporção de interessados no BCC que responderam a pesquisa é $11,9 \%$, e 44,8\% não pretendem cursar). Entre as interessadas no BCC, quando perguntado qual era o fator motivador para essa escolha de curso, as opções mais assinaladas foram "perspectivas profissionais" (84,6\%) e vocação $(76,9 \%)$, enquanto entre os homens essas mesmas opções também foram as mais citadas, porém com $60,0 \%$ e $100 \%$, respectivamente. A maior dificuldade no curso apontada por ambos os gêneros foi o alto grau de complexidade das disciplinas $(69,2 \%$ e $80 \%$ na opinião das mulheres e homens, respectivamente). Além disso, entre as mulheres, a opção "ambiente pouco acolhedor entre colegas e docentes" foi indicada $(30,8 \%)$ como uma dificuldade, sendo que apenas um homem apontou esse fator.

Outro ponto levantado pelo questionário foi "Na sua opinião, por que há poucas mulheres cursando BCC na UFABC, levando em conta o número de ingressantes no BC\&T?". Foi observado que as alternativas que apontavam questões de gênero como um fator influenciador foram as mais marcadas pelas mulheres, entre elas: i) ambiente pouco acolhedor às mulheres $(69,2 \%)$; ii) intimidação pela maioria masculina $(61,5 \%)$; iii) desestimulação de novas alunas devido à baixa presença de mulheres $(46,2 \%)$. Quando perguntado o mesmo para os homens, as respostas dadas pelo grupo se dividiu de forma similar entre todas as opções, sendo que $40 \%$ consideraram o desinteresse pelo campo de trabalho como um fator relevante, enquanto apenas $15,4 \%$ de mulheres marcaram essa opção.

\subsection{Divulgação}

$\mathrm{Na}$ era digital e das redes sociais considerou-se imprescindível que o projeto estivesse presente no âmbito digital por meio de um site, um grupo e uma página no Facebook. O site contém informações gerais sobre o projeto e nele podem ser encontrados conteúdos que são divulgados no Facebook, sendo um local onde essas informações estão 
melhor organizadas e podem ser facilmente encontradas. Desde o lançamento (8 meses), o site teve 908 usuárias(os), 1297 sessões (média de 3m04s) com 4235 page views. Também há um grupo fechado no Facebook, administrado por alunas do projeto, que atualmente conta com 175 participantes, todas mulheres. A escolha de ser um grupo só para mulheres foi tomada com base na necessidade de existir um ambiente mais acolhedor, no qual as mulheres pudessem se sentir à vontade para conversar, tirar dúvidas e compartilhar experiências, sendo um contraponto às salas de aula compostas por maioria masculina.

A página no Facebook, com 361 curtidas até fevereiro de 2019 (318 mulheres e 43 homens), foi criada para ser um meio de divulgação de atividades do projeto, ações que se alinhem ao tema mulheres na tecnologia e referências femininas no campo da Ciência da Computação. Por ser uma rede social de amplo uso, este canal possibilita alcançar os homens, cuja participação também é importante nesse debate.

Embora o Facebook tenha sido escolhido por tornar a comunicação mais rápida e facilitar o alcance de um número maior de pessoas, notou-se que há pouca interação, mesmo no grupo voltado apenas para mulheres. Por isso, outra iniciativa de divulgação realizada foi o Workshop "Mulheres na Computação: os desafios da carreira", durante a IV Semana do CMCC com o tema "Mercado \& Inovação". No encontro, a equipe de alunas apresentou o projeto, os primeiros resultados da pesquisa sobre a representatividade feminina na UFABC e divulgaram as principais ações do grupo. $\mathrm{O}$ Workshop também incluiu palestras sobre representação de gênero na tecnologia. Além disso, as palestrantes se juntaram a professoras e alunas convidadas em uma roda de conversa para troca de experiências sobre os desafios das mulheres no mercado de trabalho e na academia.

O Workshop contou com 32 participantes, que foram incentivados a interagir durante as apresentações, enviando perguntas e respondendo enquetes pelo aplicativo sli.do. As convidadas responderam às perguntas durante a roda de conversa, expondo suas vivências como mulheres inseridas na área de Tecnologia, discutindo sobre estereótipos de gênero e sobre os desafios encontrados ao longo de suas carreiras. Junto com as questões enviadas pelo aplicativo, foram recebidos comentários positivos pela iniciativa do evento, além da manifestação ao vivo de meninas e meninos que se identificaram com o tema.

A última iniciativa adotada para divulgação foi a criação de uma conta no Instagram, onde fotos e vídeos são postados em tempo real para que as pessoas acompanhem as atividades que estão sendo realizadas. Essa rede foi criada pelas alunas após perceberem que as pessoas ao seu redor passam um tempo considerável do seu dia neste aplicativo, havendo um alto engajamento das pessoas. Após 100 dias de criada a conta no Instagram, o número de seguidores(as) chegou a ser de mais de 200 pessoas.

\subsection{Ensino}

Com a baixa representatividade feminina apontada pela pesquisa e notando-se a dificuldade nas matérias iniciais da computação, identificou-se a necessidade de apoiar as alunas no primeiro contato com lógica de programação e com uma linguagem de programação. Além de incentivar as alunas que já finalizaram as matérias básicas a darem continuidade em seus estudos da área. Para isso, foi projetado o minicurso "Lógica de programação e solução criativa usando Python" com o objetivo de propiciar um ambiente favorável para desenvolver autoconfiança, aumentar a motivação das meninas e quebrar estereótipos sobre a aprendizagem do pensamento computacional.

O minicurso foi projetado usando o modelo ADDIE (acrônimo das fases Analyze, Design, Develop, Implement, Evaluate) para o desenvolvimento de materiais 
instrucionais (Morrison, 2010). Na fase de Análise realizou-se uma seleção somente de discentes mulheres da UFABC, visando uma maior identificação do grupo, para verificar se este ambiente proporciona mais cumplicidade entre as alunas a ponto de se sentirem mais confortáveis em interagir e motivadas para a aprendizagem. A seleção foi realizada por meio de um formulário, com perguntas sobre o nível de conhecimento das alunas. Das 71 meninas inscritas, 40,8\% afirmaram não ter nenhum conhecimento sobre lógica de programação, $42,3 \%$ afirmaram saber o que é lógica de programação, mas não como aplicá-la e somente $16,9 \%$ responderam que sabem o que é lógica e a programar. No processo de seleção foram priorizados os grupos que afirmaram ter nenhum ou pouco conhecimento sobre o tema. Devido à capacidade do laboratório, foram escolhidas 50 alunas, das quais 28 confirmaram a participação no minicurso, 12 tiveram conflitos de horário ou problemas pessoais e 10 não responderam. Também houve a preocupação de selecionar monitoras do BCC em estágio mais avançado do curso para uma maior identificação com as alunas.

O Projeto e Desenvolvimento do material do minicurso foi realizado inteiramente pelas monitoras com a revisão das professoras, visando abordar os conteúdos de forma lúdica. O objetivo foi demonstrar que a lógica está presente no cotidiano de todas e em vários domínios de aplicação (Gomes \& Mendes, 2007).

Para a Execução do minicurso foram reservados laboratórios da UFABC, equipados com computadores para uso individual. Utilizou-se a ferramenta VisualG para a parte de lógica de programação, pois mostrou-se mais amigável para o primeiro contato na escrita de algoritmos (Mannila, Peltomäki \& Salakoski, 2006). Na segunda parte, optou-se pela linguagem de programação Python, utilizando a plataforma de codificação online Repl.it, para aprofundar as habilidades de programação. Nas aulas expositivas, foram abordados exemplos da vida cotidiana e resolvidos exercícios simples em conjunto entre monitoras e alunas. Na parte prática, problemas com maior grau de dificuldade foram resolvidos individualmente, com a supervisão e apoio das monitoras. O minicurso teve duração de nove encontros de duas horas cada. O minicurso iniciou com 28 alunas e teve apenas duas desistências.

Para a fase de Avaliação, foi projetado e aplicado um segundo questionário online, este de forma anônima, para verificar o nível de satisfação das alunas, com os mesmos parâmetros do questionário de inscrição. Das 13 alunas que responderam o questionário final, 92,3\% afirmaram saber o que é lógica de programação e como aplicá-la, enquanto 7,7\% afirmaram conhecer lógica de programação, mas ainda não saber como aplicá-la. Esse último formulário também incluiu perguntas para verificar se o ambiente somente formado por mulheres fez diferença para o aprendizado das alunas, bem como sobre a confiança das meninas quanto a área de programação. Como apresentado na Figura 3, 92,3\% das alunas responderam que sentiram que faz diferença uma turma composta apenas por mulheres; e 76,9\% afirmaram estar confiantes sobre a área de programação, e demonstraram interesse em aprender mais sobre o assunto. Por fim, no formulário foi perguntado sobre o material desenvolvido para as aulas pelas monitoras que ministraram o curso. Das 13 meninas que responderam, 92,3\% afirmaram que o material estava claro e fácil de compreender e $7,7 \%$ afirmaram que o material poderia ser melhorado. $\mathrm{O}$ material e recursos utilizados no laboratório se mostraram eficazes para o grupo de alunas selecionadas. 
Sobre o fato de ser um curso só para mulheres

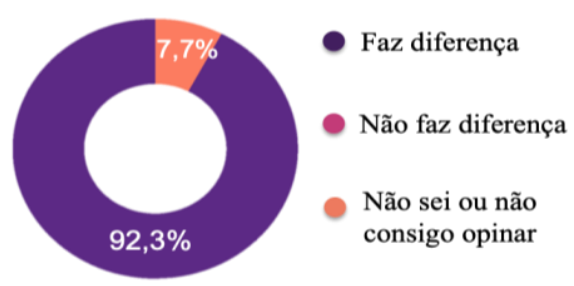

Como você se sente quanto a área de programação

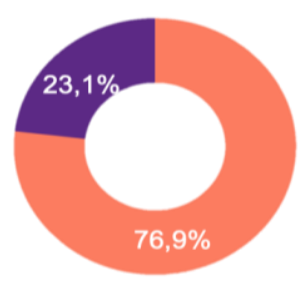

Figura 3. Exemplos de perguntas do questionário de satisfação e respostas.

Ao final do minicurso foi realizada uma atividade extra, com a participação de 12 meninas, com um teste para avaliação do conhecimento adquirido, uma roda de conversa e uma confraternização (Figura 4). O teste foi realizado com o jogo de tabuleiro "Guerra em Alto Mar" (Oliveira, 2017). O jogo contém perguntas de lógica de programação e Python, que exploram conhecimentos de variáveis, entrada e saída de dados, operadores lógicos e matemáticos, estruturas de repetição e condição, e funções. Foram realizados dois jogos simultâneos de uma equipe contra outra, com três meninas cada. Na pontuação total das quatro equipes, houve um acerto de $88 \%$ das questões. A maior parte dos erros ocorreram em perguntas que haviam detalhes que passaram despercebidos, apesar de algumas meninas relatarem que as perguntas eram muito fáceis. No mesmo dia, a equipe organizou uma roda de conversa com a Camila Sass, aluna no último ano de graduação no BCC, fundadora do grupo Mulheres na Computação UFABC e membro da equipe do $++C \&$ TpM. Ela contou do seu início na universidade, sobre o BCC e sua trajetória e experiências na área de TI. Por fim, houve uma confraternização de encerramento com as alunas, monitoras e professoras.
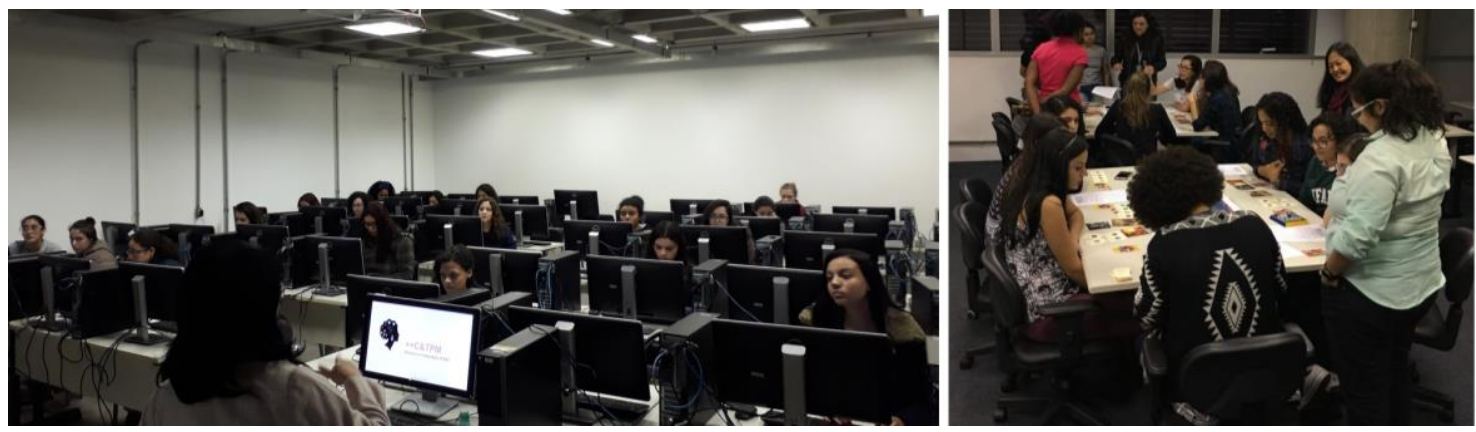

Figura 4. Aula (esquerda) e atividade de avaliação (direita) do minicurso.

Todas as alunas que participaram do minicurso relataram que não obtiveram alfabetização computacional no ensino fundamental e médio. Também afirmaram que seria muito importante ter esta aprendizagem antes do ingresso à Universidade, para que pudessem ter conhecimento sobre a computação e enxergá-la como uma opção universitária. Além disso, devido a participação no minicurso, elas pensam no BCC como possível escolha de curso pós BC\&T. A Figura 5 mostra parte dos comentários registrados pelas alunas no formulário de avaliação final do curso, que ressaltam a importância da identificação, do estímulo e confiança na aprendizagem computacional. 
A ideia como um todo foi incrivel e vocês são ótimas, parabéns por tudo. Adoraria manter contato e aprender outras linguagens. Muito obrigada s2

O curso foi ótimo, acredito que deveria ter outras edições com o mesmo conteúdo eu só achei muito curto, gostaria de me aprofundar mais e aprender outras linguagens de programação.

Gostei muito do projeto e o curso vai me ajudar a continuar meus estudos individuais sobre data science com python.

Gostaria muito que o curso continuasse, em Python. Gostei muito, gostaria de parabenizá-las

Eu adorei a iniciativa! As instrutoras foram super gentis, didáticas e receptivas. Espero que cada vez mais meninas gostem de computação e sigam na área. Estou ansiosa para novas edições com outros cursos!

Muito obrigada pelo curso!!! meu professor meio louco passou java agora no primeiro quadrimestre e não explicou nada, porém as aulas de lógica que tive no curso, me ajudaram muuuuito. Obrigada de verdade, espero que mais meninas possam ter a oportunidade de participar desse curso maravilhoso, obrigada tb à todas as meninas, por sempre explicar com carinho e de forma atenciosa!! e tenho mto interesse em outros cursos, e em outras linguagens, e etc, se tiver, irei fazer com certeza, obrigada mais uma vez!!! $<3$ ps: Erika obrigada pelos docinhos, mimou muito a gente $<3<3<3$

Muito obrigada pelo curso!!! A ideia do projeto foi incrivel, sempre quis aprender programação mas nunca soube por onde começar. Todas vocês foram muito atenciosas e ajudaram muito com todas as dúvidas, além dos mimos de chocolate em todas as aulas. Obrigada $<3$

Adoro vcs! Melhor mini-curso, espero muitos outros aaaaa

Meu profundo agradecimento pela oportunidade de aprender um pouco mais sobre esse assunto em um ambiente bem confortável

Figura 5. Alguns depoimentos registrados no questionário de satisfação.

\section{Desenvolvimento e Resultados de Iniciativas de Extensão}

$\mathrm{O}++\mathrm{C} \& \mathrm{TpM}$ surgiu como projeto de monitoria para discentes de graduação da UFABC. No entanto, a equipe de alunas e professoras percebeu que a disparidade de gêneros encontrada em universidades é um reflexo de um processo que começa cedo com a construção de estereótipos que definem atividades diferentes para meninas e meninos (Margolis \& Fisher, 2003). Muitas meninas desde pequenas não cogitam os cursos de Computação, seja por desconhecimento da área, ou por considerá-la masculina, não se vendo como apta para ocupar tal espaço (Master, Cheryan \& Meltzoff 2016). Sendo assim, é preciso promover o ensino do pensamento computacional nas escolas e discutir a pauta da necessidade de mulheres na tecnologia no ambiente familiar e escolar.

Neste contexto, o projeto estendeu suas ações para o ensino de lógica de programação com foco em alunas do ensino médio das escolas públicas da região do ABC. Em um curto período de divulgação (cinco dias) foram realizadas 302 inscrições para uma nova oferta do minicurso, das quais $80 \%$ foram mulheres e $14,5 \%$ de alunas e alunos de ensino médio. Do total, 30,8\% afirmaram não terem conhecimento na área de programação, 48,8\% sabiam o que é programação, mas não como aplicá-la, e 20,4\% indicaram que sabem conceitos de programação e como aplicá-la, tendo interesse apenas em conhecer a linguagem Python. Dos 60 homens inscritos, apenas 18,3\% afirmaram não ter conhecimento sobre programação, 58,3\% conheciam programação, mas não como aplicá-la, e 23,3\% tinham interesse apenas na linguagem Python.

Na seleção, foram priorizadas as alunas de ensino médio e da graduação e professoras e professores do ensino básico, que afirmaram não ter conhecimento em programação e as que não sabiam aplicá-la, nesta ordem de prioridade. Diferentemente da primeira versão do curso, nesta segunda oferta, os conteúdos de lógica de programação 
foram abordados junto com os conceitos básicos da linguagem Python. Por isso, somente foram realizados quatro encontros de três horas cada.

O minicurso foi inicialmente projetado para oferecer 40 vagas. No entanto, a equipe decidiu abrir mais uma turma, totalizando 60 vagas, dentre elas cinco para homens. Para isso, a equipe de monitoras, inicialmente formada por três instrutoras principais e três monitoras auxiliares, foi reforçada com mais cinco meninas participantes da primeira oferta. Concluíram o curso 33 participantes, sendo 28 mulheres, das quais cinco eram alunas de ensino médio, três professoras do ensino básico, sete discentes e uma técnica administrativa da universidade, além de outras 12 da comunidade externa.

Para avaliar o aproveitamento das(os) participantes foram realizadas atividades gamificadas usando a plataforma kahoot!. Na atividade final, 76\% das questões foram respondidas de forma correta. Para avaliar a execução do minicurso foi aplicado um formulário de satisfação. Os resultados mostraram que após o minicurso $78 \%$ dos que responderam o formulário se sentem confiantes e gostariam de aprender mais sobre programação e mais de $70 \%$ gostaria de estudar computação ou carreira similar.

Por outro lado, a equipe percebeu como necessário dar suporte àquelas alunas de graduação já matriculadas no BCC. Pela experiência das próprias monitoras, sendo minoria nas disciplinas do curso, é fácil se sentir sozinha, perdida e não pertencente a esse espaço. Por isso, o ++C\&TpM implementou um projeto de mentoria, com o intuito de conectar mulheres com mais experiência às mais novatas, para que elas recebessem suporte em questões acadêmicas, sobre carreira, mercado, organização dos estudos, etc.

Foram formadas 15 duplas com base em seus interesses, e deu-se liberdade para que cada uma determinasse o seu melhor meio de comunicação (call, e-mail, troca de mensagens, presencial). Dessa forma, as monitoras foram responsáveis por montar as duplas e fazer feedbacks mensais para acompanhar a evolução do programa. No entanto, ao realizar o feedback foi perceptível que houve uma baixa participação das meninas, principalmente das mentoradas. Com o feedback foram obtidas 9 respostas de mentoras e apenas duas das mentoradas. Dentre as respostas, que englobaram 10 duplas, observouse que apenas uma dupla manteve contato, três iniciaram contato, mas esse foi perdido após algumas interações e cinco não iniciaram uma conversa.

Um ponto importante levantado pelas participantes foi que o primeiro contato seja feito em parceria com a equipe do $++\mathrm{C} \&$ TpM. Além disso, foi sugerido que ao longo da mentoria sejam realizadas ações como reuniões, palestras e rodas de conversa, que contribuam para a criação de uma rede de apoio que vá além de cada dupla e que haja espaço para dúvidas e problemas não resolvidos serem expostos, aumentando as chances de solução.

\section{Conclusões e Trabalhos Futuros}

Este artigo apresentou um projeto voltado para engajar meninas no curso de Ciência da Computação na UFABC alinhado com os objetivos ODS 4 e 5 da Agenda 2030 para o Desenvolvimento Sustentável da ONU. Nele foi realizada uma pesquisa sobre o desempenho das alunas nas disciplinas básicas de tecnologia e se ele afetaria seus interesses pela área de computação, refutando essa hipótese. Além disso, foram apresentadas estratégias de divulgação que serão ampliadas para abordar mais modelos femininos na computação, relatar experiências e aplicações práticas, bem como atingir um maior número de pessoas. Por fim, cursos de ensino de pensamento computacional foram oferecidos para alunas da graduação e do ensino médio da região. Acreditamos que as ações de divulgação e ensino atingiram um público de mais de 200 meninas. Todas as 
atividades do projeto (incluindo a escrita deste artigo) foram protagonizadas pela equipe de oito alunas com a ajuda de cinco professoras. Os resultados também foram apresentados em um seminário de pesquisa para professoras(es) e estudantes de graduação e pós-graduação da Computação, com cerca de 50 presentes.

O projeto foi novamente aprovado no edital de monitoria de 2019. Na nova edição pretende-se fomentar as ações de mentoria, baseadas na promoção de interação mensal entre alunas veteranas e ingressantes, seguindo uma abordagem similar à proposta por Besana e Dettori (2004). O objetivo é estimular a autoconfiança e ajuda mútua através de ações de aconselhamento, monitoria e apoio nas adversidades e desafios. Além disso, serão consideradas as sugestões de melhorias reportadas na primeira implementação do programa de mentoria, pelas alunas, professoras e participantes. Espera-se, com essa iniciativa, colocar em prática ideais como "juntas, somos mais fortes" e que o empoderamento feminino pode ser construído de forma conjunta entre as mulheres, além de desmistificar a ideia de rivalidade feminina. As pesquisas serão aprofundadas para avaliar de forma quantitativa e qualitativa a evolução do cenário de gênero do BCC e do BC\&T em geral, bem como o impacto do ++C\&TpM. Também pretende-se promover discussões abertas ao público interno e externo sobre o tema de desigualdade de gênero nas exatas e tecnologias.

Como trabalhos futuros, espera-se fomentar o ensino da lógica de programação nas escolas da região com foco no público feminino. Isso pode contribuir para reverter os dados apresentados anteriormente, visto que aprender essas habilidades durante a formação escolar contribui para que as meninas se sintam mais seguras e à vontade com o universo da computação (UNESCO, 2017). Dessa forma, elas podem se sentir mais preparadas para entrarem em cursos superiores relacionados a essa área. Para isso, nesta nova edição do projeto, o minicurso será ministrado como curso de extensão aberto a meninas de ensino médio e graduação, bem como para professoras e professores da rede de ensino básico da região. O contato das estudantes de ensino médio com a Universidade, em especial com alunas de graduação, pode contribuir para a quebra de estereótipos e o aumento do número de meninas ingressantes que pretendam cursar Ciência da Computação e carreiras afins.

$\mathrm{O}$ desenvolvimento do projeto demandou o esforço conjunto de alunas e professoras. Espera-se que a nova edição permita a consolidação da metodologia que possa ser aplicada no nível institucional. O objetivo é definir políticas e iniciativas da universidade para aumentar a porcentagem de mulheres em outros cursos de Ciência e Tecnologia com baixa representatividade feminina, como por exemplo, Matemática, Física e as Engenharias da Informação e de Instrumentação, Automação e Robótica.

\section{Referências}

BANERJEE, M., SCHENKE, K., LAM, A., \& ECCLES, J. (2018). The Roles of Teachers, Classroom Experiences, and Finding Balance: A Qualitative Perspective on the Experiences and Expectations of Females Within STEM and Non-STEM Careers. International Journal of Gender, Science And Technology, 10 (2), 287-307.

BERTON L., \& dos SANTOS J.A.A. (2018). Gender differences in technologies courses at ICT-UNIFESP Brazil. 10th LAWCC.

BESANA, G., \& DETTORI, L. (2004). Together is better: strengthening the confidence of women in computer science via a learning community. Journal of Computing Sciences in Colleges, 19(5), 130-139. 
BIM, S., FIGUEIREDO, K., \& MACIEL, C. (2017). Por Mulheres na Computação no Brasil: análise das ações e publicações do evento WIT. 9th LAWCC.

BLANEY, J.M., \& STOUT, J.G. (2017). Examining the relationship between introductory computing course experiences, self-efficacy, and belonging among firstgeneration college women. In: Proceedings of the ACM SIGCSE Technical Symposium on Computer Science Education. 69-74.

CHERYAN, S., MASTER, A., \& MELTZOFF, A.N. (2015). Cultural stereotypes as gatekeepers: increasing girls' interest in computer science and engineering by diversifying stereotypes. Frontiers in Psychology, 6.

DASGUPTA, N., \& STOUT, J. G. (2014). Girls and women in science, technology, engineering, and mathematics: STEMing the tide and broadening participation in STEM careers. Policy Insights from the Behavioral and Brain Sciences, 1(1), 21-29.

da SILVA, E.A.A., DE OLIVEIRA, S.C.M., BERENGUEL, O.L, GIANCOLI, A.P.M., \& de SOUZA, T. (2018). Promovendo a Participação de Mulheres nos cursos de Exatas do IFSP, campus Bragança Paulista. 10th LAWCC.

dos SANTOS, W.O. (2017). Mulheres na Computação: Uma Análise da Participação Feminina nos Cursos de Licenciatura em Computação. Workshop da Licenciatura em Computação, CBIE.

ELSEVIER. (2017). Gender in the Global Research Landscape. Recuperado em janeiro de 2019, de https://www.elsevier.com/_data/assets/pdf_file/0008/265661/Elsevier GenderReport_final_for-web.pdf.

FREITAS, R. D.; NAKAMURA, F.; SANTOS, T.M.; LOBO, L.; LAUSCHNER, T.; \& MACHADO, A.L. (2017). Undergraduate women in Computing: where did they come from, how are they and where they are going? 9th LAWCC.

GOMES, A., \& MENDES, A. J. (2007). Learning to program-difficulties and solutions. In International Conference on Engineering Education-ICEE, 7.

GOOGLE. (2018). Google diversity annual report 2018. Recuperado em janeiro de 2019, de https://static.googleusercontent.com/media/diversity.google/pt-BR//static/ pdf/Google_Diversity_annual_report_2018.pdf.

GROSSI, M.G.R., BORJA, S.D.B., LOPES, A.M., \& ANDALÉCIO, A.M.L. (2016). As mulheres praticando ciência no Brasil. Revista Estudos Feministas, 24(1), 11-30.

HERRMANN, S.D., ADELMAN, R.M., BODFORD, J.E., GRAUDEJUS, O., OKUN, M.A., \& KWAN, V.S. (2016). The effects of a female role model on academic performance and persistence of women in STEM courses. Basic and Applied Social Psychology, 38(5), 258-268.

HOlAndA, M., DANTAS, M., COUTO, G., CORREA, J. M., ARAúJO, A.P.F., \& WALTER, M.E.T. (2017). Perfil das Alunas no Departamento de Computação da Universidade de Brasília. 11th WIT, CSBC.

HUGHES, R. M., NZEKWE, B., \& MOLYNEAUX, K. J. (2013). The single sex debate for girls in science: A comparison between two informal science programs on middle school students' STEM identity formation. Research in Science Education, 43(5).

IBGE. (2018). Estatísticas de gênero: indicadores sociais das mulheres no Brasil. Coordenação de População e Indicadores Sociais. Estudos e pesquisas. Informação demográfica e socioeconômica, 38. 
KOZIEVITCH, N., BERARDI, R., BIM, S.A., PEREIRA, P., \& RANGEL, J. (2018). A presença de mulheres no corpo discente do curso de Sistemas de Informação da UTFPRCuritiba. 10th LAWCC.

LEWIS, C.M, ANDERSON, R.E., \& YASUHARA, K. (2016). "I Don't Code All Day": Fitting in Computer Science When the Stereotypes Don't Fit. In Proc. ACM Conference on International Computing Education Research (ICER '16).

MACIEL, C., \& BIM, S.A. (2017). Programa Meninas Digitais - ações para divulgar a Computação para meninas do ensino médio. Anais do Computer on the Beach, 327-336.

MAIA, M.M. (2016). Limites de gênero e presença feminina nos cursos superiores brasileiros do campo da computação. Cadernos Pagu. n. 46, pp. 223-244.

MANNILA, L., PELTOMÄKI, M., \& SALAKOSKI, T. (2006). What about a simple language? Analyzing the difficulties in learning to program. Computer Science Education, 16, 211-227.

MARGOLIS, J., \& FISHER, A. (2003). Unlocking the clubhouse: Women in computing. MIT Press.

MASTER, A., CHERYAN, S., \& MELTZOFF, A.N. (2016). Computing whether she belongs: Stereotypes undermine girls' interest and sense of belonging in computer science. Journal of Educational Psychology, 108(3), 424-437.

MATOS, E.S., CORLETT, E.F., FERREIRA, A.C.C., \& dos SANTOS, J.M.O. (2017). Sobre a trajetória de professoras negras na pós-graduação em Ciência da Computação: alguns resultados preliminares. In 11th WIT, CSBC.

MERVIS, J. (2011). Weed-out courses hamper diversity. Science, 334(6061), 1333.

MICROSOFT CORP. (2017). Why Europe's girls aren't studying STEM. Microsoft Philanthropies. Recuperado em janeiro de 2019, de http://bit.ly/2qiFT5u.

MONARD M.C., \& FORTES R.P. (2013). Uma visão da participação nos cursos de Ciência da Computação no Brasil. 6th LAWCC.

MONTEIRO R.S., MARINHO J.M.P., BRAGA R.B., VIANA M.N., \& de OLIVEIRA C.T. (2017). Delineando o Perfil Feminino Discente do Bacharelado em Ciência da Computação do IFCE campus Aracati. 11th WIT, CSBC.

MOREIRA, J., MATTOS, G., BARRETO, L., CAVACO, I., \& MOREIRA, R. (2016). Atraindo Meninas para a Ciência da Computação: Métodos e Ferramentas. Workshop de Ensino em Pensamento Computacional, Algoritmos e Programação, CBIE.

MORRISON, G.R. (2010). Designing Effective Instruction, 6th ed. John Wiley \& Sons.

NAKAMURA F., LOBO L., FREITAS R., ALMEIDA T., MACHADO A.L., \& LAUSCHNER T. (2017). Participação feminina em cursos de computação: um estudo no Instituto de Computação da Universidade Federal do Amazonas. 11th WIT, CSBC.

NUNES, M.A.S.N., LOUZADA, C.S., SALGUEIRO, E.M., ANDRADE, B.T., LIMA, P.S., \& FIGUEIREDO, R.M.C.T. (2016). Mapeamento de iniciativas brasileiras que fomentam a entrada de mulheres na computação. 10th WIT, CSBC.

PROPLADI. (2017). Pesquisa, censo e opinião discente UFABC (2010-2016). $\begin{array}{lllll}\text { Recuperado em de } & \text { janeiro }\end{array}$ http://propladi.ufabc.edu.br/images/perfil_aluno/perfil_discente_2016.pdf. 
OLIVEIRA, R.N.R. (2017). Guerra em Alto Mar: Relatando a Construção de um Jogo de Tabuleiro para a Área de Educação em Computação. TCC, UFPB, Rio Tinto.

POSSER, C.V., \& TEIXEIRA, A.C. (2016). Mulheres que aprendem informática: Um estudo de gênero na área de TI. Workshop de Informática na Escola, CBIE

REINKING, A., \& MARTIN, B. (2018). The Gender Gap in STEM Fields: theories, Movements, and Ideas to Engage Girls in STEM. Journal of New Approaches in Education Research, 7(2), 148-153, 2254-7339.

ROSENTHAL, L., LONDON, B., LEVY, S.R., \& LOBEL, M. (2011). The roles of perceived identity compatibility and social support for women in a single-sex STEM program at a co-educational university. Sex Roles: A Journal of Research, 65(9-10), 725736.

SAX, L., BLANEY, J., LEHMAN, K., RODRIGUEZ, S., GEORGE, K., \& ZAVALA, C. (2018). Sense of Belonging in Computing: The Role of Introductory Courses for Women and Underrepresented Minority Students. Social Sciences, 7(8), 122.

SBC. (2017). Educação Superior em Computação, Estatísticas - 2017. Recuperado em janeiro de 2019, de http://www.sbc.org.br/documentos-da-sbc/category/133-estatisticas.

SCHMIDT A.E., BRITTO H., \& KOEHLER M. (2016). Levantamento e análise dos dados da presença feminina discente no curso de Bacharelado em Sistemas de Informação do Instituto Federal Catarinense, Campus Camboriú. 10th WIT, CSBC.

UNESCO. (2018). Decifrar o código: educação de meninas e mulheres em ciências, tecnologia, engenharia e matemática (STEM). Recuperado em janeiro de 2019, de http://www.unesco.org/new/pt/brasilia/about-this-office/single-

view/news/portuguese_version_of_cracking_the_code_girls_and_womens.

UFABC. (2017). Projeto Pedagógico Institucional. Recuperado em janeiro de 2019, de http://www.ufabc.edu.br/images/imagens_a_ufabc/projeto-pedagogico-institucional.pdf.

WING, J. M. (2006). Computational thinking. Communications of the ACM, 49(3). 